\title{
PERSISTENT CURRENT AND PERSISTENT CHARGE IN
}

\section{NANOSTRUCTURES}

\author{
I.O.KULIK \\ Department of Physics, Bilkent University \\ Bilkent 06533, Ankara, Turkey
}

\begin{abstract}
Quantum effects in the interaction of electromagnetic field with small, but not microscopically small, metallic particles and rings are investigated. The aspects of mesoscopic systems related to time-independent (or magnetic) Aharonov-Bohm effect, time-dependent (electric) Aharonov-Bohm effect, and to quantum high-frequency effects in a coupled system: optical fiber+ mesoscopic conducting loop are discussed.
\end{abstract}

\section{Introduction}

Electromagnetic field is primarily a wave. The quantum mechanics tell us that the field exists in a form of quanta, the photons.

Electrons are believed to be primarily particles, the point-like objects. Quantum physics then introduces a wave aspect of electron through the notion of particle-wave dualism. The electrons and photons are to be considered on same footing.

Unlike photons, electrons are charged, which brings a new aspect to the wave mechanics of electrons interacting with the electromagnetic field. This was first recognized by Aharonov and Bohm [1] who have shown that electromagnetic potentials $\mathbf{A}, \phi$ are of primary importance, rather than electromagnetic fields $\mathbf{H}, \mathbf{E}$ themselves, and that at certain topology of space (or space-time) it may appear that the effects related to vector potential $\mathbf{A}$ alone (with $\mathbf{H}$ and $\mathbf{E}$ identically equal to zero), or that of scalar potential $\phi$ alone (again, with $\mathbf{H} \equiv 0, \mathbf{E} \equiv 0)$ may exist. These effects are: the persistent currents in metallic loops $[2,3]$, persistent charges in metallic granules [4] and resistance oscillations in mesoscopic rings and networks $[5,6]$.

We will consider the wave phenomena in collection of electrons which are large in number (say, $N \sim 10^{10}$ in a metallic granule of size $\sim 1 \mu \mathrm{m}$ ) but at certain condition may behave similar to a single atom. The condition is specified by the requirement that electron in a granule does not suffer inelastic collision which acts as a measuring event, resulting in the reduction of the wave packet and elimination of coherent electron phase. [The elastic scattering, on contrary, preserves the electron phase.] The phase-breaking length of electron

\footnotetext{
${ }^{1}$ Also at: B. Verkin Institute for Low Temperature Physics and Engineering, Acad. Sci. of Ukraine, Lenin ave. 47, Kharkov 310164, Ukraine.
} 
$l_{\varphi}$ is related to the electron-electron and electron-phonon scattering as

$$
l_{\varphi}^{-1} \sim l_{\text {inel }}^{-1} \sim \frac{1}{v_{F}}\left(\lambda_{e-e} \frac{T^{2}}{\varepsilon_{F}}+\lambda_{e-p h} \frac{T^{3}}{\omega_{D}^{2}}\right)
$$

in a clean metal $\left(l_{\text {elast }} \gg L\right)$, and

$$
l_{\varphi} \sim\left(l_{\text {inel }} l_{\text {elast }}\right)^{1 / 2}
$$

in a dirty metal $\left(l_{\text {elast }} \ll L\right)$ where $l_{\text {inel }}, l_{\text {elast }}$ are inelastic and elastic mean free paths, $L$ is the typical size of the system, $\omega_{D}$ the Debye energy, and $v_{F}$ and $\varepsilon_{F}$ are the electron Fermi velocity and Fermi energy, respectively. $\lambda_{e-e}$ and $\lambda_{e-p h}$ are dimensionless electronelectron and electron-phonon coupling constants which can be crudely taken as quantities of order 1. Typically, $\varepsilon_{F} \sim 1-10 \mathrm{eV}$ and $\omega_{D} \sim 10-100 \mathrm{meV}$ which means that mesoscopic behavior appears in system of micron size at temperature $T$ below $1 K$.

\section{Persistent current}

Persistent current is a dissipationless non-decaying current in a mesoscopic loop induced by a static magnetic flux piercing the loop (Fig.1). The current was first predicted for a normal-metal loop in the paper [2] following the pioneering work of Imry and Gunther [7] who have shown that in a one-dimensional superconducting loop with a superconductivity destroyed by quantum fluctuations, a non-decaying, or "persistent" current (according to later terminology [3]) reappears . As stated in Ref.[2], in a normal metal “...the current

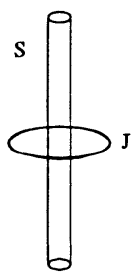

(a)

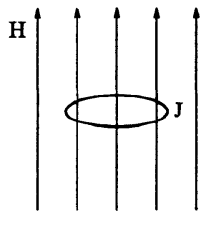

(b)

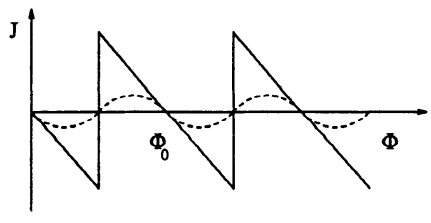

(c)

Figure 1. (a) Schematic of the Aharonov-Bohm effect in the ring with a solenoid, and, (b) in an homogeneous magnetic field; (c) The dependence of current on magnetic flux. Solid line corresponds to $T=0$, dotted line to $T>0$.

state corresponds to a minimum of free energy, so that allowance for dissipation does not lead to its decay".

The origin of the current in a ring can be understood with a generic Hamiltonian

$$
H=-t \sum_{n=1}^{N}\left(a_{n}^{+} a_{n+1} e^{i \alpha}+a_{n+1}^{+} a_{n} e^{-i \alpha}\right) .
$$

where $a_{n}$ is an electron annihilation operator at site $n, t$ is the transmission amplitude between the nearest sites, and $\alpha$ is the phase related to the magnetic flux in the ring

$$
\alpha=2 \pi \frac{\Phi}{N \Phi_{0}}, \quad \Phi_{0}=\frac{h c}{e} .
$$


The quantity $\Phi_{0}=4 \cdot 10^{-7} G \cdot \mathrm{cm}^{2}$ is the flux quantum of a normal metal. The flux in the ring determined as

$$
\Phi=\int \mathbf{A} d \mathbf{l}=\int \mathbf{H} d \mathbf{S}
$$

can be produced either by a solenoid inserted inside the ring (Fig.1(a)), or by an externally applied magnetic field (Fig.1(b)). In the first case, no classical effect of magnetic field is expected because for the electrons confined within the ring, the magnetic field appears to be identically zero. The effect of vector potential is introduced by the phase factor $\exp (i \alpha)$ in the hopping amplitude in Eq.(3) where $\alpha=\int_{n}^{n+1} \mathrm{~A} d \mathrm{l}$. Calculating the eigenstates of the electron within the Hamiltonian of Eq.(3)-

$$
\varepsilon_{k}=-2 t \cos (k+\alpha), \quad k=\frac{2 \pi}{N} m, m=0,1,2 \ldots N-1
$$

and calculating the thermodynamic potential

$$
\Omega=-T \sum_{k} \ln \left(1+e^{-\beta\left(\varepsilon_{k}-\mu\right)}\right)
$$

where $\beta=1 / T$ and $\mu$ is the chemical potential, we evaluate a current $J$ as a derivative

$$
J=-c \frac{\partial \Omega}{\partial \Phi}
$$

The dependence $\Omega(\Phi)$ vanishes in the limit $N \rightarrow \infty$ consistent with the van Leuven theorem stating that in classical mechanics thermodynamic parameters are independent of $\mathbf{A}$ and $\phi$. Calculation shows that $J$ is periodic in $\Phi$ with a period of flux quantum $\Phi_{0}$, see Fig.1(c).

The oscillatory dependence is a hallmark of the persistent-current effect and serves as an experimental indication that the effect is there [8,9]. The magnitude of the persistent current

$$
J_{c} \sim e v_{F} / L \sim 10^{2} n A \text { at } T=0 \text { and } L \sim 1 \mu m
$$

where $L=N a$ is the circumference of the ring ( $a$ is atomic period). Estimate (9) corresponds to a current produced by one electron orbiting around the ring with velocity of order of Fermi velocity $v_{F} \sim 10^{8} \mathrm{~cm} / \mathrm{s}$.

The magnetic moment corresponding to this current $M=\frac{1}{c} J S$ where $S$ is the cross section of the ring, is much larger than microscopic Bohr magneton

$$
M / \mu_{B} \sim k_{F} L \sim 10^{4} .
$$

However, the magnetic energy associated with the moment, $E_{1} \sim M^{2} / L^{3}$, is much smaller than electron level spacing $\Delta \varepsilon \sim \hbar v_{F} / L$,

$$
E_{1} / \Delta \varepsilon \sim \frac{e^{2} / a}{m c^{2}} \sim 10^{-5}
$$

which means that we may neglect the self-action of the current on the magnetic field within the ring. 
The current remains nonzero if scattering (both elastic and inelastic), $3 d$ lattice effects and finite temperature are taken into account. However, the magnitude of critical current reduces.

Inelastic scattering reduces the magnitude of $J_{c}$ according to an exponential factor $\exp \left(-L / l_{\varphi}\right)$. The $3 d$ effects increase the value of $J_{c}$ by a factor of $\sqrt{N_{\perp}}$ where $N_{\perp}=k_{F}^{2} S / 4 \pi^{2}$ is the number of "perpendicular channels" (number of components of electron momenta perpendicular to the direction of current). The effect of finite temperature is to mix the electron states around the Fermi energy and therefore to reduce the value of $J_{c}$ because due to $k$ dependence of the energy of individual electron states (6), the $\alpha$-dependence of $\Omega$ flattens at increasing $T$. The effect is accounted for by a factor $\exp \left(-L / \xi_{T}\right)$ [2] where $\xi_{T}$ is the temperature dependent "coherence length" of normal metal

$$
\xi_{T}=\hbar v_{F} / 2 \pi T \text {. }
$$

[In a dirty metal, $\xi_{T}$ is given by $\xi_{T}=\left(\hbar v_{F} l_{\text {elast }} / 2 \pi T\right)^{1 / 2}$. ]

The effect of elastic collision on magnitude of the persistent current is more subtle. It seems at first that elastic scattering does not influence $J_{c}$ since it preserves the coherent phase of electron. The other extreme is to introduce the uncertainty of the energy due to scattering $\delta \varepsilon \sim \hbar v_{F} / l_{\text {elast }}$ and to compare that with the energy spacing in presence of vector potential $\Delta \varepsilon \sim \hbar v_{F} / L$. The reduction factor of order $l_{\text {elast }} / L$ may follow [10] which however is not correct. Kirczenow [11] showed that smaller reduction does in fact emerges in a specific model of potential scattering.

If we introduce some barriers $V_{i}$ representing impurities in ring than at large $V_{i}$ the $\Phi$ dependent part of energy will be proportional to $1 /\left|V_{i}\right|$, whereas resistivity due to these barriers will increase as $\left|V_{i}\right|^{2}$. This means that $J_{c} \sim \Delta \Omega$ should scale with $1 / \sqrt{R}$ and not with $1 / R$ as follows from the reduction factor $l_{\text {elast }} / L$.

Consider perfect $1 d$ ring interrupted at some point with a $\delta$-functional barrier of height $V$. Eigenvalue problem in the tight-binding approximation (3) is easily solved giving for the current from a particular eigenstate (6) labeled with $m$, an expression

$$
j_{m}=(-1)^{m} \frac{e m^{2}}{2 \pi V} \sin 2 \pi \frac{\Phi}{\Phi_{0}} .
$$

Summing over all $k_{m}$ up to $\left|k_{m}\right|=k_{F}$ gives an estimate of $J_{c}$

$$
J_{c} \sim \frac{e v_{F}}{L} \cdot\left|\frac{t^{\prime}}{t}\right|
$$

where $t^{\prime}$ is the transmission amplitude in the ring with a barrier.

Considering more general case of many barriers and combining the efect of many transverse channels we receive an estimate of the maximal current in dirty metal

$$
J_{c} \sim \frac{e v_{F}}{L}\left(\frac{R_{0}}{R}\right)^{1 / 2}
$$

where $R_{0}$ is quantum of resistance $R_{0}=h / 2 e^{2} \simeq 12.9 k \Omega$ and $R$ is the reristance along the ring (resistance of a rod of length $L$ and cross section $S$ received by cutting the ring at some point). The formula (15) is in qualitative agreement with the experimentally measured magnitude of persistent current [8]. 


\section{Persistent charge}

Consider two small (mesoscopic) pieces of metal $P_{1}, P_{2}$ separated by distance $d$ and placed either in the field of time-dependent scalar potential $\phi(t)$, with electric field confined within a capacitor (Fig.2(a)), or in an external electric field producing the scalar potential difference between the particles $\phi=E d$ (Fig.2(b)). In both cases, the quantity in question

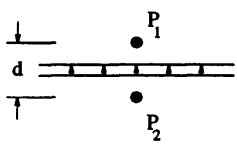

(a)

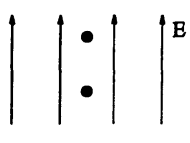

(b)

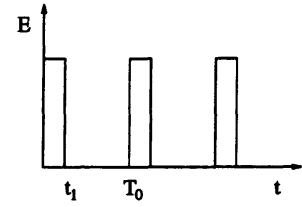

(c)

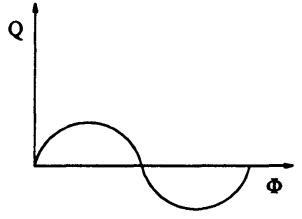

(d)

Figure 2. Schematic of the electric Aharonov-Bohm effect with (a) a thin capacitor, and, (b) in an homogeneous electric field. (c) and (d) represent $E(t)$ and $Q(\Phi)$ dependences respectively.

which determines the Aharonov-Bohm effect is the "electric flux" $\Phi^{\prime}$ defined as

$$
\Phi^{\prime}=\int E d x d t
$$

Integral is taken over the period $\left(T_{0}\right)$ of the electric field variation. If, for instance, we choose the dependence $E(t)$ in the form of the Kronig-Penney barrier (Fig.2(c))

$$
E(t)= \begin{cases}E_{0}, & \text { if }\left|t-n T_{0}\right|<t_{1} \\ 0, & \text { if }\left|t-n T_{0}\right|>t_{1}\end{cases}
$$

then we receive $\Phi^{\prime}=E_{0} t_{1} d$. The generic Hamiltonian of the problem

$$
H=\frac{e \phi(t)}{2} \sigma_{z}+T_{12} \sigma_{x}
$$

where $\sigma_{i}$ are Pauli matrices and $T_{12}$ is the hopping amplitude between $P_{1}$ and $P_{2}$.

Suppose that at $t=0$ the system acquired amplitudes $u_{0}, v_{0}$ in the upper and lower states of (18). Then, at a later time, it may occupy any of two states with amplitudes $u$, $v$ thus creating the dipole moment $P=e d\left(|u|^{2}-|v|^{2}\right)$. Solution of the time-dependent Schrödinger equation gives

$$
P(t)=4 e \operatorname{Re}\left(u_{0}^{*} v_{0}\right) \operatorname{Im} \zeta(t)
$$

where

$$
\zeta(t)=\frac{e^{2 \pi i \nu}-e^{2 \pi i N \nu}}{1-e^{2 \pi i \nu}}+F e^{2 \pi i N \nu}, \quad \nu=\frac{e V_{0}}{h}=\frac{\Phi^{\prime}}{\Phi_{0}},
$$

and $N=\left[t / T_{0}\right], \quad F=\left\{t / T_{0}\right\}$ where $[x]$ and $\{x\}$ are the integer and the fractional parts of $x$, respectively. The quantity $\nu$ is the electric flux $\Phi^{\prime}$ in units of flux quantum (4).

The charge $Q=P / e$ is accumulated between the granules. The value of the charge is an oscillating function of $\Phi^{\prime} / \Phi_{0}$, analogous to the oscillation of persistent current vs $\Phi / \Phi_{0}$ in a static Aharonov-Bohm effect. 
If the electric flux $\Phi^{\prime}$ is slowly varying in time (with period $T_{1}$ much larger than $T_{0}$ ) then we will observe the periodic variation of $Q$ with $\Phi^{\prime}$ provided that $T_{1}$ is much smaller than the phase-breaking time $\tau_{\varphi}=l_{\varphi} / v_{F}$. The oscillating behavior will persist to temperature of order of $\hbar / T_{0}$. No such experiment have sofar been performed.

\section{Resistance oscillation}

Suppose that mesoscopic loop of Fig.1(a) is connected by conducting wires with two thermal reservoirs $R_{1}, R_{2}$ held at different voltages $V_{1}, V_{2}$ (Fig.3(a)). Then the transport

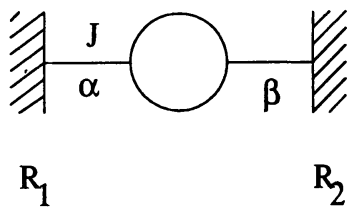

(a)

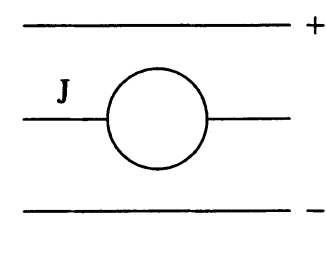

(b)

Figure 3. (a)Mesoscopic loop connected to incoherent voltage sources (thermal reservoirs) $R_{1}, R_{2}$. (b)A "quantum mechanical transistor" [13].

current flowing through the loop will be superimposed over the persistent current produced by the external flux. The electrical resistance of the loop $R=\left(V_{2}-V_{1}\right) / J_{t r}$ can be calculated with the Landauer formula $[6,12]$

$$
R=\frac{2 e^{2}}{h} \sum_{\alpha \beta}\left|t_{\alpha \beta}\right|^{2}
$$

where $t_{\alpha \beta}$ is the transmission amplitude between one of perpendicular channels of electron eigenstate to the left of the ring $(\alpha)$ and to the right of one $(\beta)$. This formula is applicable when electron state $\alpha$ enters the ring from the left equilibrium reservoir, and emerges to the state $\beta$ in the right reservoir. It is assumed that energy conserves within the ring, i.e. motion of electron is "ballistic" in energy. Magnetic flux $\Phi$ piercing the ring will affect electronic states $\alpha, \beta$ and therefore will alter the resistance of the ring.

Resistance oscillation are found in many electric measurements on mesoscopic rings (see [5] and refererences therein). The temperature, size, and purity dependence of resistance oscillation are similar to those of a persistent current. Typical magnitude of conductance variation is of order of $e^{2} / h$. An example of $R(\Phi)$ dependence is shown in Fig.4.

In a loop configuration shown in Fig.3(b), an additional electric field was applied [13] perpendicular to the direction of the transport current (the "control gate" of "quantum mechanical transistor") resulting in the shift of oscillation pattern of Fig.4 proportional to the gate voltage. The possible explanation of the experiment may be in considering various classical paths of electron within the ring (Fig.5).

In the dirty ("diffusive") regime $l_{\text {elast }} \ll L$, various local loops of electron trajectory may be formed inside the ring. These loops change their energy in a random way when 

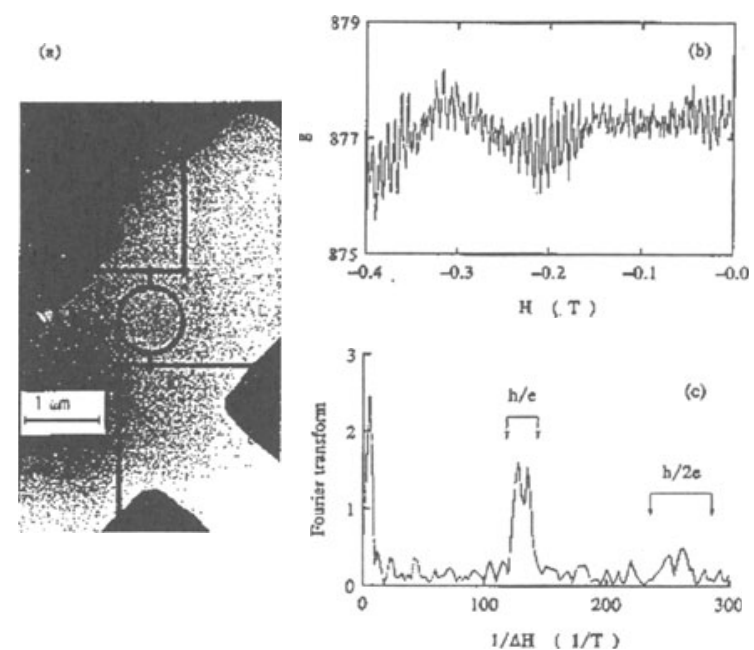

Figure 4. Resistance oscillation in the loop of gold. (a)Loop configuration; (b) $R$ vs $H$ dependence; (c)Fourier transform of $R(H)$ showing maximum at flux quantum periodicity. From Ref.[5].

magnetic flux $\Phi$ changes, causing the electron redistribution between the loops and, as a result, the shift in the oscillation pattern $R(\Phi)$. The effect is reduced in magnitude because of screening of electric field inside the metal. Quite large voltages on the control gate $\left(v_{G} \sim 1 V\right)$ are required to see the substantial shift in the interference pattern $R(\Phi)$, consistent with this reduction.

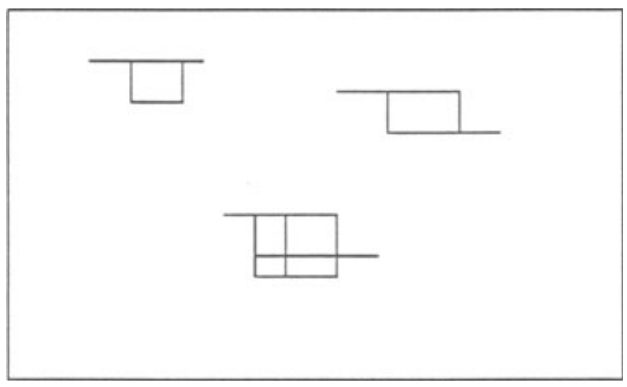

Figure 5. Random network of local loops inside the disordered conductor. Changing of magnetic field causes electron redistribution between the loops.

\section{Quantum interference in high frequency field}

We now turn to another configuration of the Aharonov-Bohm experiment with mesoscopic rings [14]. Suppose that nanoscale loop encloses an optical fiber with the high frequency 
field pumped through it (Fig.6). If the static magnetic field $H_{d c}$ is simultaneously applied

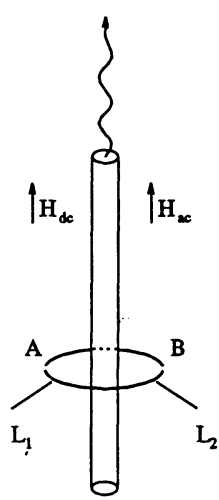

(a)

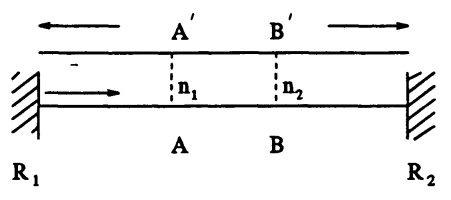

(b)

Figure 6. (a)The mesoscopic loop $(L)$ enclosing an optical fiber. In the $T E_{01}$ mode, the magnetic field $H_{a c}$ is aligned parallel to external field $H_{d c}$ and to fiber axis.

(b)Schematic of weakly coupled loop with weak links at points $A, B$ coupled to thermal reservoirs $R_{1}, R_{2}$.

along the fiber axis, resistance of the loop will periodically change not only with the static flux $\Phi=H_{d c} S$ but also with the amplitude of a.c. power in the fiber. Most strong effect is expected in the $T E_{01}$ mode of the fiber in which magnetic field $H_{a c}$ has component along the fiber axis (Fig.7). We assume that loop is "weakly connected", i.e. has narrow regions

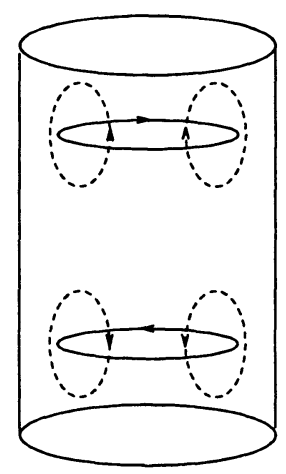

Figure 7. Field configuration in the $T E_{01}$ mode of optical fiber. Solid lines are lines of force of magnetic field, dotted lines the lines of force of electric field.

$A, B$ such that an a.c. field is concentrating near the latter. The size of the loop should be smaller than few wavelengths of optical field to ensure that total a.c. flux within the loop is not equal to zero.

The generic Hamiltonian corresponding to the setup of Fig.6(b) can be represented in the 
form

$$
\begin{aligned}
H & =-t_{0} \sum_{n}\left(a_{n}^{+} a_{n+1}+b_{n}^{+} b_{n+1}\right)+H_{i n t}, \\
H_{i n t} & =-t_{1} a_{n_{1}}^{+} b_{n_{1}} e^{i \alpha_{1}}-t_{2} a_{n_{2}}^{+} b_{n_{2}} e^{i \alpha_{2}}+\text { h.c. }
\end{aligned}
$$

where the first term accounts for the hopping along two independent chains $\left(a_{n}, b_{n}\right.$ are the electron annihilation operators in the chains), whereas the second one ascribes the interaction between the chains. The lower chain is connected to two thermal reservoirs $R_{1}$, $R_{2}$ for measurement of the resistance variation with the a.c. and d.c. fields.

Hopping amplitudes between the chains are modulated in phase due to static and alternating fields according to $t_{i} e^{i \alpha_{i}}$ with

$$
\alpha_{i}=\alpha_{i}^{0}+A_{i} \sin \left(\omega t+\delta_{i}\right)
$$

The phase difference $\alpha_{1}^{0}-\alpha_{2}^{0}$ is controlled by a static flux

$$
\alpha_{1}^{0}-\alpha_{2}^{0}=\frac{2 \pi \Phi_{d c}}{\Phi_{0}}, \quad \Phi_{d c}=\int \mathbf{B} d \mathbf{S}, \quad \Phi_{0}=\frac{h c}{e}
$$

whereas the time-dependent parts relate to an a.c. power in fiber.

Solution of Hamiltonian (22) can be achieved by perturbation over $t_{1}, t_{2}$ in the frequency domain $\hbar \omega>4\left|t_{0}\right|$ in which inelastic transitions corresponding to change in energy $n \hbar \omega$ are forbidden. Hamiltonian (22) does not have unperturbed states outside the bandwidth of the one-dimensional metal $4\left|t_{0}\right|$.

Employing the identity

$$
e^{i z \sin \varphi}=\sum_{n=-\infty}^{\infty} J_{n}(z) e^{i n \varphi}
$$

where $J_{n}(z)$ are Bessel functions of order $n$, we can decompose $H_{\text {int }}$ into the Fourier series

$$
H_{\text {int }}=\sum_{n=-\infty}^{\infty} H_{i n t}^{(n)} e^{i n \omega t}
$$

with

$$
H_{i n t}^{(n)} \equiv-t_{1} e^{i \alpha_{1}^{0}} J_{n}\left(A_{1}\right) a_{n_{1}}^{+} b_{n_{1}}-t_{2} e^{i \alpha_{2}^{0}} J_{n}\left(A_{2}\right) a_{n_{2}}^{+} b_{n_{2}} .
$$

Coupling between the plane-wave states of the unperturbed Hamiltonian

$$
\psi_{k}=\sum_{n} e^{i k n} a_{n}^{+}|0\rangle, \quad \phi_{k}=\sum_{n} e^{i k n} b_{n}^{+}|0\rangle
$$

results in transition between the chains with probability

$$
W_{ \pm}\left(\psi_{k} \leftrightarrow \phi_{ \pm k}\right)=\left|\left\langle\phi_{ \pm k}\left|H_{i n t}^{(0)}\right| \psi_{k}\right\rangle\right|^{2} /\left|v_{k}\right|
$$

where subscript "+" refers to transition $\psi_{k} \rightarrow \phi_{-k}$ and "-" to transition $\phi_{k} \rightarrow \psi_{-k}$. Since momentum $|k|$ is conserved at transition, we may introduce kinetic equations for the 
populations $f_{1}^{ \pm}$of electrons with momenta $k$ and $-k$ in the lower chain, and similarly $f_{2}^{ \pm}$ in the upper chain. Taking into account symmetry between the transitions $\psi_{k} \rightarrow \phi_{ \pm k}$ and $\phi_{k} \rightarrow \psi_{ \pm k}$ we receive

$$
\begin{aligned}
& \frac{d f_{2}^{-}}{d t}=-W_{0} f_{2}^{-}\left(1-f_{1}^{-}\right)-W_{-} f_{2}^{-}\left(1-f_{1}^{+}\right)+W_{0} f_{1}^{-}\left(1-f_{2}^{-}\right)+W_{-} f_{1}^{+}\left(1-f_{2}^{-}\right) \\
& \frac{d f_{2}^{+}}{d t}=-W_{0} f_{2}^{+}\left(1-f_{1}^{-}\right)-W_{0} f_{2}^{+}\left(1-f_{1}^{+}\right)+W_{+} f_{1}^{-}\left(1-f_{2}^{+}\right)+W_{0} f_{1}^{+}\left(1-f_{2}^{+}\right)
\end{aligned}
$$

in the upper chain, and

$$
\begin{aligned}
& \frac{d f_{1}^{-}}{d t}=Q_{+}-W_{0} f_{1}^{-}\left(1-f_{2}^{-}\right)-W_{+} f_{1}^{-}\left(1-f_{2}^{+}\right)+W_{0} f_{2}^{-}\left(1-f_{1}^{-}\right)+W_{+} f_{2}^{+}\left(1-f_{1}^{-}\right) \\
& \frac{d f_{1}^{+}}{d t}=Q_{-}-W_{-} f_{1}^{+}\left(1-f_{2}^{-}\right)-W_{0} f_{1}^{+}\left(1-f_{2}^{+}\right)+W_{-} f_{2}^{-}\left(1-f_{1}^{+}\right)+W_{0} f_{2}^{+}\left(1-f_{1}^{+}\right)
\end{aligned}
$$

in the lower chain where $Q_{ \pm}$is the influx of electrons from the reservoirs

$$
Q_{ \pm}=\frac{v_{k}}{L}\left(f_{1}^{ \pm}-f_{0}\left(\varepsilon_{k} \pm \frac{e V}{2}\right)\right)
$$

and $f_{0}(\varepsilon)=(\exp (\beta(\varepsilon-\mu))+1)^{-1}$ is the equilibrium Fermi distribution.

In a steady-state, $d f / d t=0$, Eq.(29) gives

$$
f_{2}^{-}=\frac{W_{0} f_{1}^{-}+W_{-} f_{1}^{+}}{W_{0}+W_{-}}, f_{2}^{+}=\frac{W_{0} f_{1}^{+}+W_{+} f_{1}^{-}}{W_{0}+W_{+}} .
$$

In the lower chain, solution of Eq.(30) at small $W$ 's gives

$$
\begin{aligned}
& f_{1}^{-} \simeq f_{0}\left(\varepsilon_{k}-e V / 2\right)+\left[W_{0} f_{2}^{-}+W_{+} f_{2}^{+}-\left(W_{0}+W_{+}\right) f_{0}\left(\varepsilon_{k}-e V / 2\right)\right] /\left|v_{k}\right| \\
& f_{1}^{+} \simeq f_{0}\left(\varepsilon_{k}+e V / 2\right)+\left[W_{0} f_{2}^{+}+W_{-} f_{2}^{-}-\left(W_{0}+W_{-}\right) f_{0}\left(\varepsilon_{k}+e V / 2\right)\right] /\left|v_{k}\right| .
\end{aligned}
$$

The current flowing between the reservoirs is

$$
J=\int_{0}^{\pi} v_{k}\left(f_{1}^{+}-f_{1}^{-}\right) \frac{d k}{2 \pi} .
$$

After simple manipulation we receive

$$
J=\int_{0}^{\pi} \frac{d k}{2 \pi} W(0)\left[\frac{W(k)}{W(0)+W(k)}+\frac{W(-k)}{W(0)+W(-k)}\right]\left[f_{0}\left(\varepsilon_{k}-\frac{e V}{2}\right)-f_{0}\left(\varepsilon_{k}+\frac{e V}{2}\right)\right] .
$$

where

$$
W(k)=t_{1}^{2} J_{0}^{2}\left(A_{1}\right)+t_{2}^{2} J_{0}^{2}\left(A_{2}\right)+2 t_{1} t_{2} J_{0}\left(A_{1}\right) J_{0}\left(A_{2}\right) \cos (\alpha+2 N k) .
$$

Conductance of the system $G=d J / d V$ is represented as $G_{0}+G_{1}$ where $G_{1}$ is the interference term proportional to $\left|t_{i}\right|^{2}$

$$
G_{1}=\frac{e^{2} t^{2} X_{0}}{2 h T} \int_{0}^{\pi} \frac{X_{0}\left[X_{+}(k)+X_{-}(k)\right]+X_{+}(k) X_{-}(k)}{\left[X_{0}+X_{+}(k)\right]\left[X_{0}+X_{-}(k)\right]} \frac{d k}{\left|v_{k}\right| \cosh ^{2}\left(\varepsilon_{k}-\mu\right) / 2 T} .
$$


In this expression

$$
\begin{aligned}
X_{s}(k)=A+B \cos (\alpha+s k L), \quad s & =-1,0,+1 \\
A=t_{1}^{2} J_{0}^{2}\left(A_{1}\right)+t_{2}^{2} J_{0}^{2}\left(A_{2}\right), B=2 t_{1} t_{2} J_{0}\left(A_{1}\right) J_{0}\left(A_{2}\right), t & =\sqrt{t_{1}^{2}+t_{2}^{2}} .
\end{aligned}
$$

where $L=2 N a$ is the total length of the ring.

Conductance of the ring is a function of both static magnetic flux $\Phi_{d c}$ and the amplitude of an a.c. field $A_{a c}=$ const $\cdot \sqrt{P}$ where $P$ is an optical power in a fiber.

The dependence $G\left(\Phi_{d c}\right)$ is similar to oscillations of static resistance discussed in Sect.4. The dependence $G(P)$ shown in Fig.8 is similar to the amplitude oscillation in the a.c. Josephson effect in superconductors $[15,16]$. The temperature variation of both oscillation

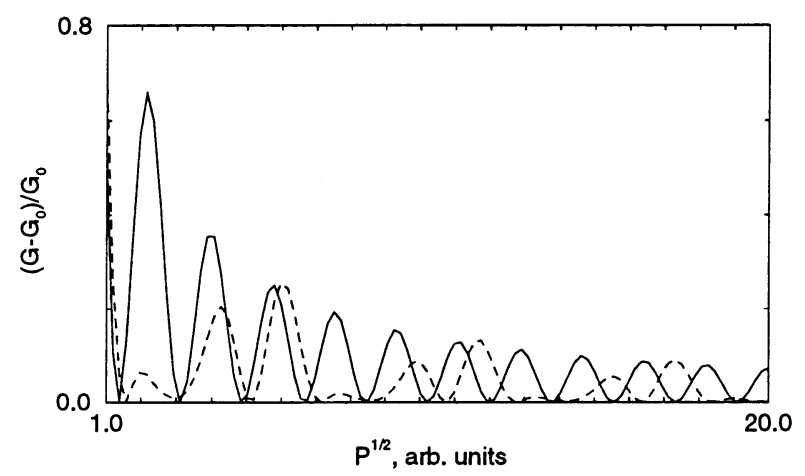

Figure 8. d.c. conductance of the loop as a function of an a.c. power. Solid line corresponds to $A_{1}=A_{2}$, the dashed line to $A_{1}=0.5 A_{2}$.

dependences is somewhat different from the temperature dependence of the amplitude of static oscillations. Formerly, the oscillation resulted from the $\Phi$ dependence of the electron states (6). Integration with respect to $k$ eliminated the oscillating component. In case of an a.c. interference, the transition probability (a coefficient before $f_{0}^{+}-f_{0}^{-}$in Eq. (35)) does not vanish after the integration with respect to $k$. Therefore the oscillation will have a nonexponential small amplitude at temperature larger than the level spacing $\Delta \varepsilon=\hbar v_{F} / L$.

\section{Other Aharonov-Bohm effects}

In recent years, there has been an interest in extension of the Aharonov-Bohm effects to systems other than metals $[17,18]$, to solid cylinders $[19,20]$ and antidots [21], to unhomogeneous magnetic fields with radial [22] or azimuthal [23] components. Aharonov and Casher [24] have considered the interaction of electron spin with electrically charged rods. The effect results in the shift of $\mathrm{AB}$ oscillation. Being quite small, Aharonov-Casher effect is enhanced in semiconductors with strong spin-orbit coupling and small effective mass of electrons [25]. Actually, such effects are nothing else than the manifestation of spin-orbit interaction. [26] which shifts the $R(\Phi)$ dependence but do not reveal the full period of oscillation like in conventional (static) AB experiment. Interaction of particle spin with electrically charged body is can be considered in context of "Berry phase" in quantum 
mechanics [27]. These and similar AB effects are reviewed in a paper [28].

\section{References}

1. Y. Aharonov and D. Bohm, Phys. Rev. 115, 485 (1959).

2. I. O. Kulik, JETP Lett. 11, 275 (1970).

3. M. Buttiker, Y. Imry, and R. Landauer, Phys. Lett. A 96, 365 (1983).

4. I. O. Kulik, Physica C, Cond. Matt. (Amsterdam) 218, 252 (1996).

5. S. Washburn, in Mesoscopic Phenomena in Solids, p.1. Eds. B.L. Altshuler, P.A. Lee, and R.A. Webb (North-Holland, Amsterdam, 1991).

6. Y. Imry, in Directions in Condensed Matter Physics, p.101. Eds G. Grinstein and E. Mazenko (World Scientific, Singapore, 1986).

7. L. Gunther, and Y. Imry, Sol. St. Communs. 7, 1391 (1969).

8. V. Chandrasekhar, R.A. Webb, M.J. Brady, M.B. Ketchen, W.J. Gallagher, and A. Kleinsasser, Phys. Rev. Lett. 67, 3578 (1991).

9. D. Mally, C. Chapelier, and A. Benoit, Phys. Rev. Lett. 70, 2020 (1993).

10. H. F. Cheung, E. K. Riedel, and Y. Gefen, Phys. Rev. Lett. 62, 587 (1989).

11. G. Kirczenow, J. Phys.: Condens. Matter 7, 2021 (1995).

12. R. Landauer, Phil. Mag. 21, 863 (1970).

13. S. Washburn, H. Schmid, D. Kern, and R. A. Webb, Phys. Rev. Lett. 59, 1791 (1987).

14. I. O. Kulik, and A. S. Shumovsky, Appl. Phys. Lett., to appear Nov. 1996.

15. I. O. Kulik, and I. K. Yanson, Josephson Effect in Superconductive Tunneling Structures (Israel Program for Scientific Translations, Jerusalem, 1972).

16. A. Barone, and G. Paterno, Physics and Applications ot the Josephson Effect (Wiley, N.Y., 1982).

17. I. O. Kulik, A. S. Rozhavsky, and E. N. Bogachek, JETP Lett., 47, 302 (1988).

18. E. N. Bogachek, I. V. Krive, I. O. Kulik, and A. S. Rozhavsky, Sov. Phys. JETP 70, 863 (1990).

19. E. N. Bogachek, and G. A. Gogadze, Sov. Phys. JETP 36, 973 (1973).

20. N. B. Brandt, E. N. Bogachek, D. V. Gitsu, G. A. Gogadze, I. O. Kulik, A. A. Nikolaeva, and Ya. G. Ponomarev, Sov. J. Low Temp. Phys. 8, 358 (1982).

21. E. N. Bogachek, and U. Landman, Phys. Rev. B 52, 14067 (1995).

22. D. Loss, P. Goldbart, and A. V. Balatsky, Phys. Rev. Lett. 65, 1655 (1990).

23. A. Stern, Phys. Rev. Lett. 68, 1022 (1992).

24. Y. Aharonov, and A. Casher, Phys. Rev. Lett. 53, 319 (1984).

25. E. N. Bogachek, and U. Landman, Phys. Rev. B 50, 2678 (1994).

26. Y. Meir, Y. Gefen, and O. Entin-Wohllman, Phys. Rev. Lett. 63, 798 (1989).

27. E. N. Bogachek, I. V. Krive, I. O. Kulik, and A. S. Rozhavsky, Mod. Phys. Lett. B 5, 1607 (1991).

28. I. V. Krive, and A. S. Rozhavsky, Int. J. Mod. Phys. B 6, 1255 (1992). 